\title{
“Chronic Periodontitis-An Oral Microbial Disease too Common?" A Short Review
}

\section{Bhandarkar GP1* and Kushal VS ${ }^{2}$}

${ }^{1}$ Department of Oral medicine and radiology, AJ Institute of Dental Sciences, India ${ }^{2}$ Department of Pedodontia, AJ Institute of Dental Sciences, India

*Corresponding author: Bhandarkar Gowri P, Reader, Department of Oral medicine and radiology, AJ Institute of Dental Sciences, Kuntikan, Mangalore-575004, Karnataka, India, Tel: +919980829341; Fax: 0824-2224968; E-mail: gowribhandarkar@gmail.com

\section{Review Article \\ Volume 2 Issue 4}

Received Date: October 30, 2017

Published Date: November 13, 2017

DOI: $10.23880 /$ oajds-16000153

\section{Abstract}

A group of diseases affecting the surrounding and supporting tissues of the teeth constitutes the periodontal disease and includes all diseases of the periodontium. Gingival inflammation, periodontal pocket formation, destruction of the periodontal ligament and alveolar bone resulting in mobile teeth symbolizes periodontitis and is accompanied by apical migration of the junctional epithelium onto the root surface. It is considered as one of the major causes of tooth loss. Thus, care must be taken to prevent this disease which leads to bone loss though a slight amount of bone loss is inevitable. This review summarizes the etiologic factors and clinical features of periodontitis. This review also presents the radiological features of periodontitis as well as the vital role of radiograph in determining the prognosis of these cases.

Keywords: Bone loss; Chronic periodontitis; Nutrient canals; Radiographs

Abbreviations: SPP: Slowly Progressive Periodontitis; CEJ: Cemento-Enamel Junction

\section{Introduction}

A group of diseases affecting the surrounding and supporting tissues of the teeth constitutes the periodontal disease and includes all diseases of the periodontium. Initially it is called gingival disease as it is confined to the gingiva and later as the supporting structures become involved, it is called periodontal disease [1]. Gingival inflammation, periodontal pocket formation, destruction of the periodontal ligament and alveolar bone resulting in mobile teeth symbolizes periodontitis and is accompanied by apical migration of the junctional epithelium onto the root surface. It is considered as one of the major causes of tooth loss [1]. Hoag \& Pawlak (1990) reported that the incidence of periodontitis is approximately one in four for adults [1]. In general, male patients have a higher incidence and severity of periodontal disease than female patients. As age progresses, periodontitis increases in prevalence and severity [2].

\section{Etiologic Factors}

Microbiological infections associated with local accrual as well as growth of bacterial plaque on teeth near the gingival margin/in the sulcus or pocket forms the chief causative feature in inflammatory periodontal disease; the tissue response being influenced by local, immune and systemic factors. Bacterial plaque is the local initiating 


\section{Open Access Journal of Dental Sciences}

factor as it causes gingival inflammation on accrual on the teeth adjacent to the gingival [2].

\section{Chronic Periodontitis}

\section{Synonyms}

Slowly progressive periodontitis (chronic adult periodontitis or chronic inflammatory periodontitis) Chronic periodontitis being the most common type of periodontitis is accompanied by plaque accrual and calculus deposits and has a slow to moderate rate of disease progression.

The following features are commonly seen in patients with chronic periodontitis.

- It is prevalent in adults but may be seen in children.

- The amount of destruction is directly proportional to local factors.

- It is accompanied by a variable microbial pattern.

- Subgingival calculus is often present.

- It develops at a slow to moderate rate and at times with rapid progression.

- It perhaps is influenced or accompanied by the following:

Systemic diseases such as diabetes mellitus/HIV infection.

Local factors predisposing to periodontitis.

Environmental factors such as cigarette smoking and emotional stress [2].

\section{Etiology}

Slowly progressive periodontitis is constantly accompanied by the presence of plaque. Copious amounts of plaque is seen in areas where there are widespread clinical changes; copious amounts of both supragingival and subgingival calculus is likewise present. Thus poor oral hygieneis associated with slowly progressive periodontitis.

Immunocompetance can differ with slowly progressive periodontitis not being accompanied by unusual systemic immunologic defects like serum, neutrophil, or monocyte abnormalities.

Periodontitis is the consequence of a complex, multifactorial collaboration between host and infective agent and is due to imbalance between the microbial insult to the attachment apparatus and the competency of the host's defense mechanisms. Local host defense factors seem to be playing a significant role in the specific pattern and severity of disease at the local site [2].
In slowly progressive periodontitis, there are no pathologic changes as a result of systemic disease.

\section{Clinical Features}

The typical features of slowly progressive periodontitis (SPP) are gingival inflammation (detected only by a periodontal probe), as a result of plaque accumulation, loss of periodontal attachment, bone loss, that leads to pocket formation. It has been frequently called chronic adult periodontitis or chronic inflammatory periodontitis as slowly progressive periodontitis takes years to progress.

The gingivae will be slightly to moderately swollen displaying color changes from a pale red to magenta. Loss of stippling accompanies blunted or rolled gingival margins as well as flattened or cratered papillae. At times, perhaps due to established low-grade inflammation, the underlying inflammatory changes may be unnoticed as a result of thickened, fibrotic marginal tissues. A periodontal probe is useful to diagnose a periodontitis lesion [2].

Spontaneous or easily provoked gingival bleeding is a common finding with inflammation-related exudates and suppuration from the pocket. An abscess may form when the pocket is sealed off, not allowing the pus to drain requiring an emergency care.

Pocket depths vary and both horizontal and angular bone loss can be detected. In advanced cases, tooth mobility is frequently seen as a result of bone loss [2].

Clinically, chronic inflammatory changes in the marginal gingiva and the presence of periodontal pockets is characteristic of slowly progressive periodontitis; radiographically, it is characterized by bone loss.

A higher incidence of infrabony pockets, angular bone loss, widening of the periodontal ligament with earlier and severe tooth mobility are detected when trauma from occlusion coexists.

Slowly progressive periodontitis is at times accompanied by regions of localized dull pain radiating deep into the jaw bone [2].

Chronic periodontitis is further classified into localized and generalized forms and categorized as slight, moderate or severe based on the following specific features:

Localized form: $<30 \%$ of sites involved Generalized form: $>30 \%$ of sites involved 


\section{Open Access Journal of Dental Sciences}

Chronic periodontitis occurs as a localized disease wherein $30 \%$ of evaluated sites demonstrate attachment and bone loss or occurs as a more generalized disease.

\section{The Disease may also be Described by Severity based on the Amount of Clinical Attachment Loss as Follows}

Mild: 2 to $4 \mathrm{~mm}$ of probing attachment loss. Minimal furcation invasions and little tooth mobility. Bleeding on gentle probing is usually detected. Radiographic evidence of bone loss is minimal (usually less than $20 \%$ of the total attachment).

Moderate: 4 to $7 \mathrm{~mm}$ of probing attachment loss. Early to moderate furcation invasions and slight to moderate tooth mobility. Bleeding on probing is common and purulence may be found. Radiographically, bone loss is usually horizontal and may consists of upto $40 \%$ of the total possible periodontal attachment on the tooth. Furcation radiolucencies may be detected.

Severe: $7 \mathrm{~mm}$ of probing attachment loss. Purulent exudates may be seen along with bleeding on probing. Significant furcation invasions, often through and through occurs. Excessive tooth mobility is also seen. Radiographic bone loss exceeds $40 \%$ and angular bony defects are found [2].

\section{Bone Destruction Caused by Extension of Gingival Inflammation}

Chronic inflammation leads to the extension of the inflammatory process to the bone and thus, the most common cause of bone destruction in periodontal disease. The inflammation from the marginal gingiva extends into the supporting periodontal tissues as gingivitis transits into periodontitis. This may be altered by the pathogenic potential of plaque/the resistance of the host (immunologic activity and other tissue-related mechanisms).

\section{General Effects of Aging}

The bone is rarified with lesser number of trabeculae and the cortical plates are thinned with reduced vascularity. Lacunar resorption is more conspicuous with increased liability to fracture. Water content of bone is diminished with age and the mineral crystals are bigger with thickened collagen fibrils [2].

\section{Alveolar Bone}

Changes in the alveolar bone are osteoporosis, diminished vascularity, reduction in metabolic rate and healing capacity. Bone resorption may be increased or decreased and the density of the bone may increase or decrease.

Barett, et al. [3] in his study, investigated the relationship between capillaropathy (studied in biopsy specimens) and alveolar bone loss (graded from the periapical roentgenogram) in the living human. He found out that with advancing age, there was progressive alveolar bone loss and also the rate of advancing bone loss with age being less in those subjects with the most physiologic capillary structure. He postulated that one of the mechanisms adding to alveolar bone loss was that the thickened capillary walls diminished delivery of nutrients to the bone [3].

\section{Radiologic Diagnosis of Periodontal Disease and Role of the Radiograph in Periodontal Disease}

The usage of the radiographs are limited in the diagnosis of periodontal disease as radiographs give a two-dimensional image of three-dimensional anatomic structures.

\section{Benefits of the radiograph}

Though it has its limitations, when viewed under good lighting conditions, a good radiograph can display most bony changes in conjunction with periodontal disease [1].

Early radiographic changes in periodontitis: Though the earliest signs of periodontal disease cannot be detected clearly in the radiograph, it still forms an indispensable part of the clinical examination. Glickman in 1972 described the following initial sequential radiographic changes that take place in periodontitis:
a) Crestal irregularities
b) Triangulation (funneling)
c) Interseptal bone changes [1]

Crestal irregularities: The mesial or distal aspect of the interdental alveolar crest appears hazy with interruption in the continuity of the lamina dura (radiopaque) forming one of the first radiographic signs of periodontitis. The extension of the inflammation into the interdental bone 
results in bone resorption. This is accompanied by widening of the vessel channels within the bone with a decrease in calcified tissue per unit of bone at the septal margin.

Triangulation (funneling): The resorption of bone along the mesial/distal aspect of the interdental (interseptal) crestal bone results in the widening of the periodontal ligament space (triangulation). The alveolar bone and root surfaces forms the sides of the triangle with the base towards the crown and the apex of the triangle towards the root. As this is an initial sign of bone degeneration, exploration for potential etiologic factors like plaque, calculus, gingivitis or food impaction is needed.

Interseptal bone changes: Periodontal disease destroys the interseptal alveolar bone thereby reducing the height of the bone. Finger-like radiolucent projections extends from the crestal bone into the interdental alveolar bone which forms one of the initial radiographic signs of periodontitis. This is due to deeper extension of the inflammation from the connective tissue of the gingiva representing widened blood vessel channels within the alveolar bone which permit the passage of inflammatory fluid and cells into the bone. This leads to a decrease in mineralized tissue per unit area. This is due to increased bone resorption along the enosteal margins of the medullary spaces produced by the inflammation extending down into the interseptal alveolar bone. It is called the "terminal stage" of chronic destructive periodontitis when all the alveolar bone support is lost and the tooth seems to "float/hang in air" on the radiograph. Reactive sclerosis seen sometimes at the border of the remaining bone is a defense mechanism by the host bone where by more bone is deposited at the borders of benign bone disease, producing a radiopaque area in the radiographic image [1].

\section{Activity of the Destructive Process}

Comparison of standardized radiographs taken over regular intervals is useful in evaluating fairly accurate destructive process of periodontal disease. The resorptive process is most likely to be active when the interdental septal bone crest is rough and irregular and the alveolar bone below the crest is bereft of bone opacity. Nutrient canals (Figures $1 \& 2$ ) point to active and rapid bone resorption. A static destructive process or slowly destructive process is suggested by a smooth surface of the alveolar bone with condensation of remaining alveolar bone in the existence of bone loss [1].
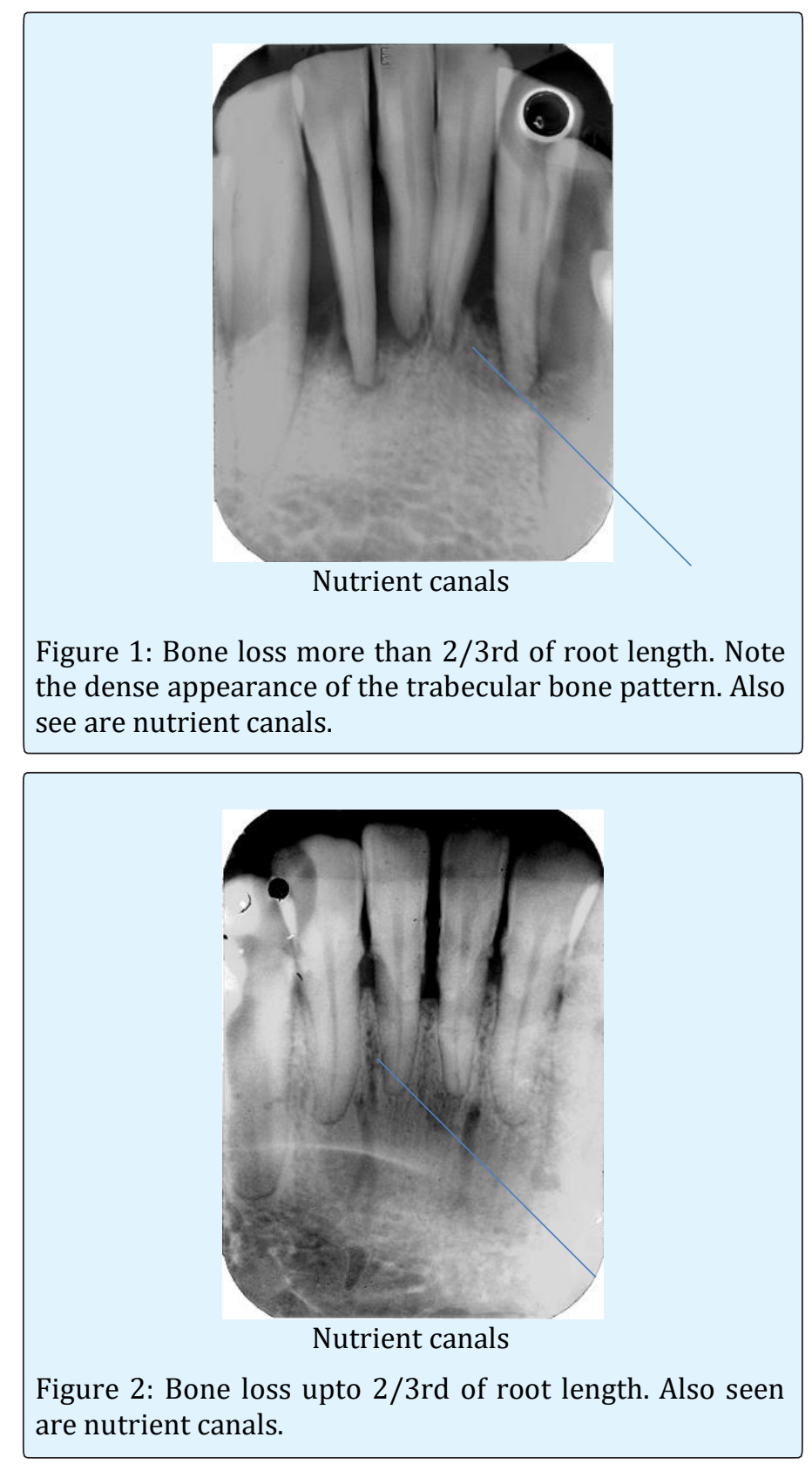

\section{Evaluation of Bone Loss}

The extent of bone loss attributed to periodontal disease is governed indirectly by the radiograph. In true sense, the radiograph determines the amount of bone remaining. The difference between the remaining coronal height and the assumed normal coronal bone level for the patients suggests the amount of bone loss. Usually, the alveolar bone height is positioned $1-1.5 \mathrm{~mm}$ apical to the cemento-enamel junction (CEJ). One of the principal 


\section{Open Access Journal of Dental Sciences}

means of assessing bone loss is made by inspecting the interproximal septal bone on the radiograph. Though, bone loss takes place on all surfaces, facial and lingual bone loss on the radiograph is masked by the thickness of the tooth. When bone loss takes place in isolated areas, it is termed as localized bone loss as opposed to generalized bone loss where the bone loss is evenly distributed all through the dental arches [1].

\section{Radiologic Features of Periodontal Disease}

\section{Bone loss and patterns of bone loss}

As the destruction of bone results in tooth loss, variations that takes place in bone are critical. The local and systemic effects regulate bone formation and bone resorption, thus maintaining an equilibrium and there by the normal height of the alveolar bone. Bone height is reduced when resorption exceeds formation. With aging, reduction in the height of the alveolar bone called as physiologic or senile atrophy takes place [2].

Linden [4] in his study found little bone destruction below the age of 40 years. In those patients aged 40 years or over, $77 \%$ of the teeth had lost at least $25 \%$ of the supporting bone. $64 \%$ of the older patients had at least one tooth with more than $50 \%$ bone loss. Only $15 \%$ of patients had more than $50 \%$ of their teeth with advanced bone loss. Women had less periodontal destruction than men [4].

\section{Radiologic Findings}

In adult periodontitis, distinctive radiographic changes are observed. On intra-oral radiographs taken with the paralleling technique, initial lesions of the interdental bone may appear fuzzy with a decrease in density of the alveolar bone. Later, bone loss is observed at the crest of the interproximal alveolar process as a result of cup-like radiolucent lesions developing interproximally. When the crest of the interdental alveolar bone extends more than 2 to $3 \mathrm{~mm}$ apical to the CEJ, alveolar bone loss occurs.
Generalized horizontal bone loss occurs when the bone loss involves most of the teeth at the same rate. Vertical bone loss is seen when the interdental alveolar bone loss develops fast at one location than another.

The prognosis is poor when the bone loss extends to the tooth apex (advanced cases of periodontitis) [2]. A thickened or widened periodontal ligament space may indicate infectious periodontitis, occlusal traumatism or tooth mobility [5].

\section{Conclusion}

Chronic periodontitis is one of the major disease leading to tooth loss. Clinical and radiologic features of periodontitis are presented. The disease should be intercepted in the early phase to prevent further bone loss in general and tooth loss in particular.

\section{References}

1. Langland OE, Langlais RP, Preece JW (2002) Principles of dental imaging. $1^{\text {st }}$ (Ed.), USA: Lippincott Williams and Wilkins pp: 357-370.

2. Carranza FA, Newman MG (1998) Clinical periodontology. $8^{\text {th }}$ (Edn.), $1^{\text {st }}$ Indian ed. Bangalore, India: W.B. Saunders Company pp: 51-55, 297-313, 326-329, 329-331.

3. Barrett RA, Cheraskin E, Ringsdorf WM (1969) Alveolar bone loss and capillaropathy. J Periodontol 40(3): 131-136.

4. Linden G (1988) Periodontal destruction and loss of the remaining natural teeth. Community Dent Oral Epidemiol 16: 19-21.

5. Bhandarkar GP (2013) A radiographic study of mandibular nutrient canals in patients with periodontal diseases. IAJD 4(1): 24-31. 